\title{
Optimal Access Point Placement for Multi-AP mmWave WLANs
}

\author{
Yuchen Liu, Yubing Jian, Raghupathy Sivakumar and Douglas M. Blough \\ Georgia Institute of Technology, Atlanta, GA, 30332
}

\begin{abstract}
mmWave communication in $60 \mathrm{GHz}$ band has been recognized as an emerging technology to support various bandwidth-hungry applications in indoor scenarios. To maintain ultra-high throughputs while addressing potential blockage problems for mmWave signals, maintaining line-of-sight (LoS) communications between client devices and access points (APs) is critical. To maximize LoS communications, one approach is to deploy multiple APs in the same room. In this paper, we investigate the optimal placement of multiple APs using both analytical methods and simulations. Considering the uncertainty of obstacles and clients, we focus on two typical indoor settings: random-obstacle-random-client (RORC) scenarios and fixed-obstacle-random-client (FORC) scenarios. In the first case, we analytically derive the optimal positions of APs by solving a thinnest covering problem. This analytical result is used to show that deploying up to 5 APs in a specific room brings substantial performance gains. For the FORC scenario, we propose the shadowing-elimination search (SES) algorithm based on an analytic model to efficiently determine the placement of APs. We show, through simulations, that with only a few APs, the network can achieve blockage-free operation in the presence of multiple obstacles and also demonstrate that the algorithm produces nearoptimal deployments. Finally, we perform ns-3 simulations based on the IEEE 802.11ad protocol at mmWave frequency to validate our analytical results. The ns-3 results show that proposed multi-AP deployments produce significantly higher aggregate performance as compared to other common AP placements in indoor scenarios.
\end{abstract}

\section{CCS CONCEPTS}

- Networks $\rightarrow$ Wireless local area networks; Network performance analysis; • Mathematics of computing $\rightarrow$ Mathematical optimization.

\section{KEYWORDS}

Millimeter wave; multi-AP; optimal placement; blockage

\section{ACM Reference Format:}

Yuchen Liu, Yubing Jian, Raghupathy Sivakumar and Douglas M. Blough. 2019. Optimal Access Point Placement for Multi-AP mmWave WLANs. In 22nd Int'l ACM Conference on Modeling, Analysis and Simulation of Wireless and Mobile Systems (MSWiM '19), November 25-29, 2019, Miami Beach, FL, USA. ACM, New York, NY, USA, 10 pages. https://doi.org/10.1145/3345768. 3355914

Permission to make digital or hard copies of all or part of this work for personal or classroom use is granted without fee provided that copies are not made or distributed for profit or commercial advantage and that copies bear this notice and the full citation on the first page. Copyrights for components of this work owned by others than ACM must be honored. Abstracting with credit is permitted. To copy otherwise, or republish, to post on servers or to redistribute to lists, requires prior specific permission and/or a fee. Request permissions from permissions@acm.org.

MSWiM '19, November 25-29, 2019, Miami Beach, FL, USA

(c) 2019 Association for Computing Machinery.

ACM ISBN 978-1-4503-6904-6/19/11 . \$ \$15.00

https://doi.org/10.1145/3345768.3355914

\section{INTRODUCTION}

With the increase in data demand and bandwidth-intensive applications, both academia and industry are pursuing new wireless technologies beyond WiFi and LTE. Millimeter wave (mmWave) communication has the potential to provide multi-gigabit per second data rates due to the large available unlicensed bandwidth $[1,2]$, and several standardization efforts such as IEEE 802.11ad and WiGig operating on $60 \mathrm{GHz}$ mmWave frequency band have already achieved per-link data rates of $7 \mathrm{Gbps}$ in indoor WLANs [3, 4].

However, mmWave signals suffer more severe path loss and penetration loss compared to lower-frequency signals. Even though the use of high-gain directional antennas can help compensate for poor propagation, it is still challenging for mmWave communication to overcome blockage effects [5]. Therefore, line-of-sight (LOS) connectivity between access points (APs) and clients becomes critical to boost link performance, and when mmWave links are blocked by obstacles such as humans and furniture, signal strength is degraded by about $30 \mathrm{~dB}$ for non-LOS paths [6], which results in lower throughput. Since the transmission ranges are very limited in a typical indoor setting, any link with LoS connectivity between an $\mathrm{AP}$ and the client is likely to have a high data rate, and maximizing the number of LOS links will also maximize network throughput.

To overcome blockages and maintain LOS paths in mmWave WLANs, three approaches are: 1) the use of reflected signals, 2) the use of relay nodes, and 3) infrastructure diversity, i.e., multiple APs. Several previous works $[7,8]$ use reflections to steer around obstacles and provide some blockage resilience, such as in [9], which proposed a solution where $60 \mathrm{GHz}$ signals can bounce off data center ceilings. However, these indirect LOS transmissions suffer severe attenuation due to the absorption of the reflecting surface [10]. Some other works [11-13] use relay nodes to maintain connectivity, and presented different relay selection algorithms. A concern with relays is the end-to-end latency increase due to the additional processing time at each hop and the relatively long-distance transmission, which may interrupt upper layer protocols. With the trend for dense AP deployments, we consider the use of multiple APs as a promising approach to address the blockage problem.

Some related works have focused on protocol design or scheduling strategy for multi-AP indoor mmWave WLANs to maintain LOS communication [14-16]. However, these works do not consider the placement problem with multiple APs, which could provide great benefits for their protocols. Intuitively, severe blockage effects can be mitigated with a good deployment strategy of APs in indoor environments, which will significantly improve the LOS performance of mmWave communication. To our knowledge, there are a few works that have considered the multi-AP deployment issue in mmWave WLANs [17, 18]. In [17], the authors proposed a heuristic algorithm that determines the locations of AP and relays to maximize the coverage by sensing reflection profile. In [18], the impact of base station deployment on LOS probability in 5G indoor scenarios was studied based on simulations, but only five special 
deployment cases were considered, where the devices followed a linear arrangement, i.e., deployed along the center line of a room.

In this paper, we study the coverage and placement of multiple APs in an indoor scenario such as Fig. 1. Due to the uncertainty of obstacles (e.g. furniture or humans) and client devices, we consider two indoor scenarios: random-obstacle random-client (RORC) scenario and fixed-obstacle random-client (FORC) scenario. In the first case without any obstacle information, the problem becomes how to deploy multiple APs to achieve optimal LOS performance in a bare room. By solving a thinnest covering problem, we derive the optimal positions of multiple APs for arbitrary-sized rectangular rooms. In FORC scenarios where obstacles are fixed and their locations/dimensions are known, e.g. the furniture has been placed in the room, we propose a shadowing-elimination search (SES) algorithm that determines multi-AP locations based on geometric analysis, and the placement of APs achieving full coverage (blockage-free) is also investigated. To evaluate the performance of multi-AP deployments in RORC scenarios, we use the ns-3 simulator based on IEEE 802.11ad protocol at mmWave frequency of 60 GHz. Through simulation results, the proposed optimal placement of APs outperforms other common AP deployment methods in both LOS probability and user throughput.

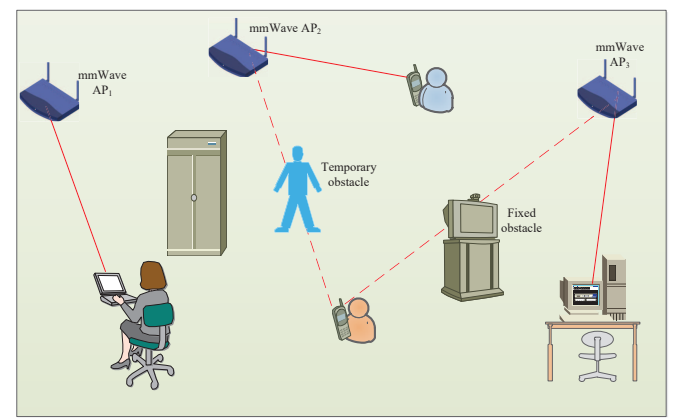

Figure 1: Multi-AP mmWave indoor wireless networks.

\section{BLOCKAGE MODEL AND MOTIVATION}

In this section, we use a stochastic geometric method to analyze blockage effects in indoor environments and evaluate the LOS performance theoretically.

From the 2-dimensional perspective, obstacles such as stationary furniture or temporarily stopped humans are assumed to form a Boolean scheme of rectangles [19]. The centers $C_{o}$ of these rectangles fall within the room and form a homogeneous Poisson point process (PPP) of density $\lambda$, where the obstacle density is defined as the expected number of obstacles in unit area. The widths $W_{o}$ and lengths $L_{o}$ are assumed to be i.i.d. distributed and follow the normal distribution as $N\left(\mu_{w}, \sigma_{w}{ }^{2}\right)$ and $N\left(\mu_{l}, \sigma_{l}^{2}\right)$, respectively. The orientation $\theta_{o}$ of every obstacle is assumed to be uniformly distributed in $(0,2 \pi]$. In this way, each obstacle $B_{o}(w, l, \theta)$ is completely characterized by the quadruple $\left\{C_{o}, W_{o}, L_{o}, \Theta_{o}\right\}$.

Based on the Boolean scheme for obstacles in 2D blockage model [20], we obtain the blockage area between client $i$ and AP $j$ as:

$$
S_{b}(w, l, \theta, r)=d_{i, j} \cdot(|\cos \theta| \cdot w+|\sin \theta| \cdot l)+w \cdot l
$$

where $d_{i, j}$ is the horizontal distance between AP and its served client, $w, l, \theta$ are the obstacle's width, length and orientation, respectively. Then, the expectation of the total number of obstacles $K$ blocking the link between client $i$ and $\mathrm{AP} j$ is derived as:

$$
\begin{aligned}
E[K] & =\int_{W_{o}} \int_{L_{o}} \int_{\Theta_{o}} \frac{1}{2 \pi} S_{b}(w, l, \theta, d) f_{W_{o}}(w) f_{L_{o}}(l) d w d l d \theta \\
& =\frac{2 d_{i, j} \cdot\left(\mu_{w}+\mu_{l}\right)}{\pi}+\mu_{w} \cdot \mu_{l}
\end{aligned}
$$

Now we introduce the height effects of obstacles and extend the blockage model to 3 dimensions. It is known that an obstacle intersecting the link between client and AP with a horizontal length of $d$ blocks the LOS path if and only if its height $h_{o}>h_{x}$, where $h_{x}=H_{A}+\frac{x}{d} \cdot\left(h_{c}-H_{A}\right)$, and $H_{A}$ and $h_{c}$ are the heights of AP and client $\left(H_{A}>h_{c}\right)$, respectively.

Since the heights of obstacles are usually different in reality, we assume that the obstacle's height $h_{o}$ follows the uniform distribution $H_{o} \sim U\left(a_{o}, b_{o}\right)$ in following parts of this paper, thus in this 3-dimensional model, each obstacle $B_{o}(w, l, h, \theta)$ is extended to be characterized by the quintuple $\left\{C_{o}, W_{o}, L_{o}, H_{o}, \Theta_{o}\right\}$. We then use $B$ to denote the event that the LOS path between the AP and the client is blocked. Assuming that the height of client $h_{c}$ also follows the uniform distributions $U\left(a_{c}, b_{c}\right)$, the conditional probability that an obstacle blocks the LOS path is:

$$
\begin{aligned}
\varepsilon & =\int_{-\infty}^{+\infty} P\left(B \mid h_{c}\right) \cdot f_{H}\left(h_{c}\right) d h_{c} \\
& =\int_{a_{c}}^{b_{c}}\left[1-\int_{0}^{1} y \cdot h_{c}+(1-y) H_{A}\right. \\
0 & =1-\frac{2 H_{A}-b_{o}-a_{o}}{2 \cdot\left(b_{c}-a_{c}\right)} \cdot \ln \left(\frac{H_{A}-a_{c}}{H_{A}-b_{c}}\right) .
\end{aligned}
$$

Note that $\varepsilon$ is independent of $K$ when the intersections with obstacles form a PPP on the LOS path between AP and client. Therefore, incorporating the heights of obstacles only introduces a constant scaling factor $\varepsilon$ to results that ignore the height. According to the PPP thinning property, $E[K]^{\prime}$ in $3 \mathrm{D}$ blockage model is $\varepsilon E[K]$ for incorporating the height effect of obstacles. Finally, we arrive at the LOS probability between client $i$ and AP $j$ :

$$
\begin{array}{r}
P_{L O S_{i, j}}=\exp \left\{-\lambda \cdot\left[\frac{2 d_{i, j}\left(\mu_{w}+\mu_{l}\right)}{\pi}-\frac{d_{i, j}\left(\mu_{w}+\mu_{l}\right)\left(2 H_{A}-b_{o}-a_{o}\right)}{\pi\left(b_{c}-a_{c}\right)} .\right.\right. \\
\left.\left.\left.\ln \left(\frac{H_{A}-a_{c}}{H_{A}-b_{c}}\right)-\frac{\mu_{w} \mu_{l}\left(2 H_{A}-b_{o}-a_{o}\right)}{2 \cdot\left(b_{c}-a_{c}\right)}\right) \cdot \ln \left(\frac{H_{A}-a_{c}}{H_{A}-b_{c}}\right)+\mu_{w} \cdot \mu_{l}\right]\right\} .
\end{array}
$$

With this analytical result, we investigate how the AP's height $H_{A}$ affects the LOS probability. According to Eq. (4), we find that $P_{L O S}$ increases monotonically with increasing $H_{A}$, which proves that the largest AP height provides the maximum LOS probability. Therefore, APs should be mounted on the ceiling of the room to achieve the best LOS performance. In what follows, we focus on 2D deployment of ceiling-mounted APs in different indoor scenarios, and the 3D coordinates of APs can be easily obtained by adding the APs' height (i.e., the room's height).

\section{MULTI-AP PLACEMENT IN RORC SCENARIOS}

In these scenarios, all obstacles and clients are randomly distributed, and the problem of maximizing LOS probability is equivalent to deploying APs so as to maximize the coverage of a given room. 


\subsection{Horizontal distance minimization}

In a specific room, we assume that clients are randomly located and will find the closest APs for connection. Thus our objective is to deploy $N$ APs in order to maximize the minimum LOS probability between each AP and its served users, and the problem can be formulated as:

$$
\max _{P \text { os }} \min _{i \in U}\left\{\max _{j \in A P} P_{L_{O S S}}\right\}, \forall P o s \in R m .
$$

where Pos are positions of APs and should not beyond the range of room. To simplify Eq. (5), we give the specific room sizes and obstacle distributions in Eq. (4), and it is observed that $P_{L O S_{i, j}}$ is inversely proportional to the horizontal distance $d_{i, j}$ between the client and AP. Therefore, we can reformulate the problem in Eq. (5) as finding APs' positions that minimize the maximum horizontal distance between the random client and its served AP, which can be described as:

$$
\underset{\text { Pos }}{\arg \min } \max _{i \in U}\left\{\min _{j \in A P} \| u_{i}-\text { Pos }_{j} \|_{2}\right\}, \forall u, \text { Pos } \in R m .
$$

Of particular note, with several specific (known) clients $u$ in the room, the RORC scenario is transformed to a random-obstaclefixed-client (ROFC) scenario, and we can find the optimal locations of APs by solving Eq. (6).

However, it is not easy to find the optimal positions of multiple APs without known users in the RORC scenario, since it will take an impractically long time to obtain the optimal solution by solving Eq. (6) when the number of APs is larger than 2.

For rectangular rooms, we can transform this multi-AP deployment (MD) problem into the Thinnest covering (TC) problem [21], which is a classical mathematical problem that aims to find $n$ congruent discs (circles) with the smallest radius $r_{n}$ that cover a specific rectangle. As Fig. 2 shows, the goal of the Thinnest covering problem is equivalent to our objective function in Eq. (6), which is trying to find optimal placement of $n$ APs that minimizes the maximum horizontal distance (referred to as the achievable distance $d_{a c}$ ) between a random client and its serving AP. Here $r_{n}$ in the TC problem is equal to $d_{a c}$ in the MD problem, and the center point of each circle in TC problem is the optimal position of each AP. In what follows, we derive the optimal placement for different numbers of APs in RORC scenarios.

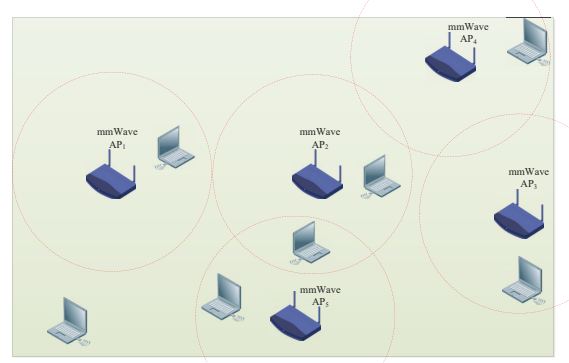

Figure 2: An example of covering a room with multiple APs.

\subsection{Multi-AP deployment cases}

In this part, we use both analytic methods and the simulated annealing approach in $[21,22]$ to solve this Thinnest covering problem, and derive the optimal locations of single or multiple APs in arbitrarysized rectangular rooms.

First, we start with the simplest case when $n$ is 1, i.e., deploying a single AP to achieve maximum LOS probability. Since ceilingmounted APs are utilized, here we just focus on 2D placement of AP in RORC indoor settings. In a specific room with the length $r_{l}$ and width $r_{w}\left(r_{l} \geq r_{w}\right)$, the thinnest covering of a rectangle with one circle is shown in Fig. 3 (a), and the optimal position for single AP is $\left(\frac{r_{l}}{2}, \frac{r_{w}}{2}\right)$. The achievable distance $d_{a c}$, i.e., the smallest radius of the circle $r_{1}$, is $\frac{1}{2} \sqrt{r_{l}^{2}+r_{w}{ }^{2}}$. This result shows that deploying the single AP in the center of the room provides the best LOS performance for randomly located clients.

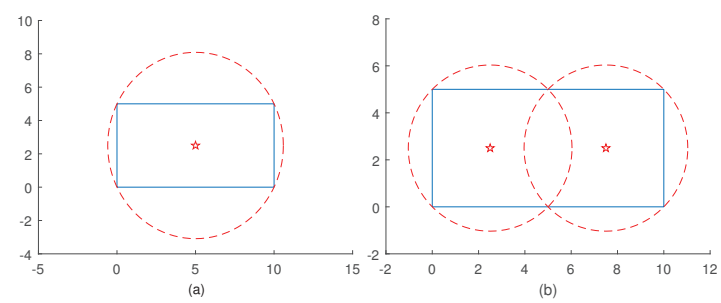

Figure 3: Optimal positions of 1-2 APs $\left(r_{l}=10 \mathrm{~m}, r_{w}=5 \mathrm{~m}\right)$.

In the 2-AP deployment case, the unique thinnest covering with two circles is shown in Fig. 3 (b), thus the optimal positions for two APs are $\left(\frac{r_{l}}{4}, \frac{r_{w}}{2}\right)$ and $\left(\frac{3 r_{l}}{4}, \frac{r_{w}}{2}\right)$, and $d_{a c}=\frac{1}{4} \sqrt{r_{l}^{2}+4 r_{w}^{2}}$.

Considering the 3-AP deployment, there are two types of thinnest covering in terms of room sizes (shown in Fig. 4). With different length-width ratios of the room, we derive the optimal positions for three APs as:

$$
\left(x_{i}{ }^{*}, y_{i}{ }^{*}\right)= \begin{cases}\left(\frac{r_{l}}{6}, \frac{r_{w}}{2}\right),\left(\frac{2 r_{l}}{3}, \frac{r_{w}}{4}\right),\left(\frac{2 r_{l}}{3}, \frac{3 r_{w}}{4}\right), & \text { if } \frac{r_{l}}{r_{w}} \leq \frac{3}{2} \\ \left(\frac{r_{l}}{6}, \frac{r_{w}}{2}\right),\left(\frac{r_{l}}{2}, \frac{r_{w}}{2}\right),\left(\frac{5 r_{l}}{6}, \frac{r_{w}}{2}\right), & \text { if } \frac{r_{l}}{r_{w}}>\frac{3}{2} .\end{cases}
$$

The corresponding $d_{a c}$ are $\frac{\sqrt{16 r_{l}^{4}+40 r_{l}^{2} r_{w}{ }^{2}+9 r_{w}{ }^{4}}}{16 r_{l}}$ and $\frac{\sqrt{r_{l}^{2}+9 r_{w}^{2}}}{6}$, respectively.
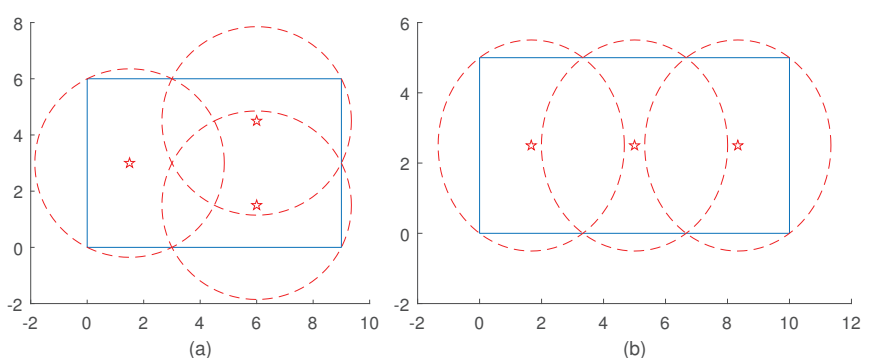

Figure 4: Optimal positions of 3 APs ((a) $r_{l}=9 \mathrm{~m}, r_{w}=6 \mathrm{~m}$; (b) $\left.r_{l}=10 \mathbf{m}, r_{w}=5 \mathbf{m}\right)$.

In the same way, the optimal positions $\left(x_{i}{ }^{*}, y_{i}{ }^{*}\right)$ for 4-AP case are derived as follow: 


$$
\left\{\begin{array}{l}
\left(\frac{r_{l}}{4}, \frac{r_{w}}{4}\right),\left(\frac{r_{l}}{4}, \frac{3 r_{w}}{4}\right),\left(\frac{3 r_{l}}{4}, \frac{r_{w}}{4}\right),\left(\frac{3 r_{l}}{4}, \frac{3 r_{w}}{4}\right) \text { if } \frac{r_{l}}{r_{w}} \leq \sqrt{\frac{5+16 \sqrt{10}}{15}} \\
\left(K_{x 1}, \frac{r_{w}}{2}\right),\left(\frac{r_{l}}{2}, 0\right),\left(\frac{r_{l}}{2}, r_{w}\right),\left(r_{l}-K_{x 1}, \frac{r_{l}}{2}\right) \text { if } \sqrt{\frac{5+16 \sqrt{10}}{15}}<\frac{r_{l}}{r_{w}}<\frac{4}{\sqrt{3}} \\
\left(\frac{r_{l}}{8}, \frac{r_{w}}{2}\right),\left(\frac{3 r_{l}}{8}, \frac{r_{w}}{2}\right),\left(\frac{5 r_{l}}{8}, \frac{r_{w}}{2}\right),\left(\frac{7 r_{l}}{8}, \frac{r_{w}}{2}\right) \quad \text { if } \frac{r_{l}}{r_{w}} \geq \frac{4}{\sqrt{3}},
\end{array}\right.
$$

where $K_{x 1}=\sqrt{\frac{r_{w}{ }^{2}}{36}\left(2 \sqrt{\frac{r_{l}{ }^{2}}{r_{w}{ }^{2}}+3}-\frac{r_{l}}{r_{w}}\right)^{2}-\frac{r_{w}{ }^{2}}{4}}$. The corresponding $d_{a c}$ are $\frac{\sqrt{r_{l}^{2}+r_{w}^{2}}}{4}, \frac{2 \sqrt{r_{l}^{2}+3 r_{w}^{2}}-r_{l}}{6}$ and $\frac{\sqrt{r_{l}^{2}+16 r_{w}^{2}}}{8}$, respectively. As Fig. 5 shows, there are 3 optimal deployment types for 4-AP case with respect to different length-width ratios of the room.

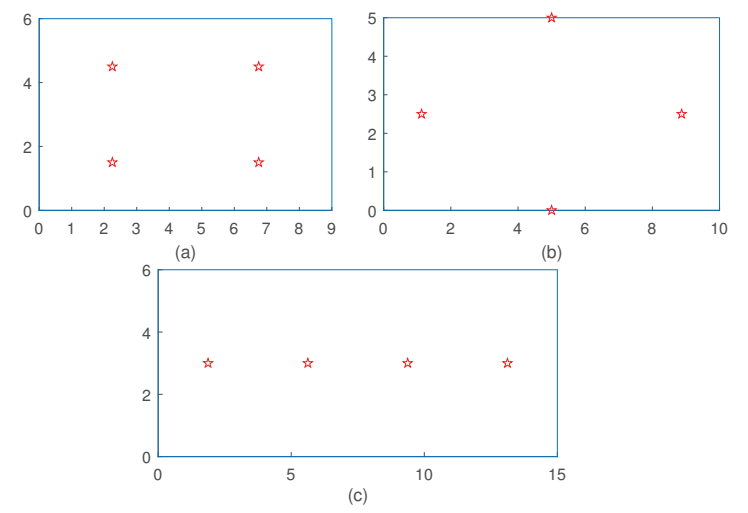

Figure 5: Optimal positions of 4 APs ((a) $r_{l}=9 \mathbf{m}, r_{w}=6 \mathbf{m}$; (b) $r_{l}=10 \mathbf{m}, r_{w}=5$; (c) $\left.r_{l}=15 \mathbf{m}, r_{w}=6 \mathbf{m}\right)$.

Considering the 5-AP deployment, the situation becomes more complicated since there are four types of thinnest covering in terms of different length-width ratios of room (shown in Fig. 6), where the optimal positions $\left(x_{i}{ }^{*}, y_{i}{ }^{*}\right)$ of these 5 APs are derived as:

$$
\begin{aligned}
& \begin{array}{c}
\text { Type I: }\left(\frac{r_{l}}{6}, K_{y 1}\right),\left(\frac{r_{l}}{2}, K_{y 1}\right),\left(\frac{5 r_{l}}{6}, K_{y 1}\right),\left(\frac{r_{l}}{4}, K_{y 2}\right),\left(\frac{3 r_{l}}{4}, K_{y 2}\right), \\
\text { if } \frac{r_{l}}{r_{w}} \leq 2.02 ;
\end{array} \\
& \text { Type II : }\left(K_{x 2}, \frac{r_{w}}{4}\right),\left(K_{x 2}, \frac{3 r_{w}}{4}\right),\left(\frac{r_{l}}{2}, \frac{r_{w}}{2}\right),\left(r_{l}-K_{x 2}, \frac{r_{w}}{4}\right) \text {, } \\
& \left(r_{l}-K_{x 2}, \frac{3 r_{w}}{4}\right), \quad \text { if } 2.02<\frac{r_{l}}{r_{w}} \leq 2.35 ; \\
& \text { Type III : }\left(K_{x 3}, \frac{r_{w}}{2}\right),\left(K_{x 4}, 0\right),\left(K_{x 4}, r_{w}\right),\left(K_{x 5}, \frac{r_{w}}{2}\right) \text {, } \\
& \left(r_{l}-K_{x 3}, \frac{r_{w}}{2}\right), \quad \text { if } 2.35<\frac{r_{l}}{r_{w}} \leq \frac{5}{\sqrt{3}} ; \\
& \begin{array}{c}
\text { Type IV: }\left(\frac{r_{l}}{10}, \frac{r_{w}}{2}\right),\left(\frac{3 r_{l}}{10}, \frac{r_{w}}{2}\right),\left(\frac{r_{l}}{2}, \frac{r_{w}}{2}\right),\left(\frac{7 r_{l}}{10}, \frac{r_{w}}{2}\right),\left(\frac{9 r_{l}}{10}, \frac{r_{w}}{2}\right), \\
\text { if } \frac{r_{l}}{r_{w}}>\frac{5}{\sqrt{3}} .
\end{array}
\end{aligned}
$$

where

$$
\left\{\begin{array}{l}
K_{y 1}=\sqrt{D_{5}(1)^{2}-\frac{r_{l}{ }^{2}}{36}}, K_{y 2}=r_{w}-\sqrt{D_{5}(1)^{2}-\frac{r_{l}{ }^{2}}{16}}, \\
K_{x 2}=\sqrt{D_{5}(2)^{2}-\frac{r_{w}{ }^{2}}{16}}, K_{x 4}=2 \sqrt{D_{5}(3)^{2}-\frac{r_{w}{ }^{2}}{4}}+D_{5}(3), \\
K_{x 3}=\sqrt{D_{5}(3)^{2}-\frac{r_{w}{ }^{2}}{4}}, K_{x 5}=3 \sqrt{D_{5}(3)^{2}-\frac{r_{w}{ }^{2}}{4}}+2 D_{5}(3),
\end{array}\right.
$$

where $D_{5}(i)(1 \leq i \leq 4)$ is the achievable distance $d_{a c}$ of Type $i$, and $D_{5}(1)$ is the smallest positive real root of the equation in Theorem 5 of [21], $D_{5}(2)=\frac{1}{6} \sqrt{5 r_{l}^{2}-2 r_{l} \sqrt{4 r_{l}^{2}-9 r_{w}^{2}}}, D_{5}(3)=$ $\frac{1}{16}\left(3 \sqrt{r_{l}^{2}+8 r_{w}^{2}}-r_{l}\right)$, and $D_{5}(4)=\frac{1}{10} \sqrt{r_{l}^{2}+25 r_{w}^{2}}$.
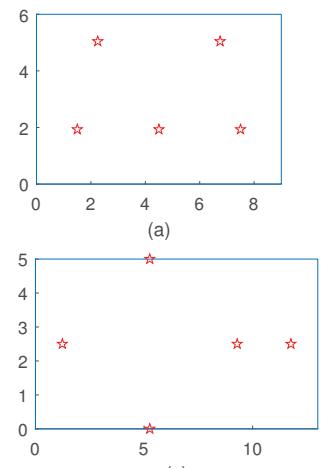

(c)
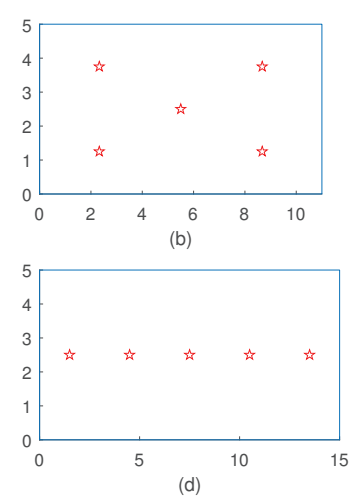

(d)
Figure 6: Optimal positions of 5 APs ((a) $r_{l}=9 \mathrm{~m}, r_{w}=6 \mathrm{~m}$; (b) $r_{l}=11 \mathrm{~m}, r_{w}=5$; (c) $r_{l}=13 \mathrm{~m}, r_{w}=5 \mathrm{~m}$; (d) $\left.r_{l}=15 \mathrm{~m}, r_{w}=5 \mathrm{~m}\right)$.

In the 6-AP deployment case, we also have four different types which are shown in Fig. 7, and the optimal positions of these 6 APs are derived as follows:

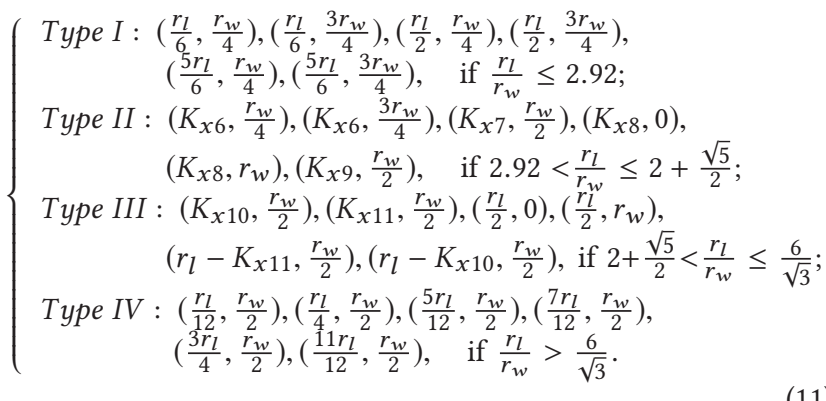

where

$$
\left\{\begin{array}{l}
K_{x 6}=\sqrt{D_{6}(2)^{2}-\frac{r_{w}^{2}}{16}}, K_{x 7}=2 \sqrt{D_{6}(2)^{2}-\frac{r_{w}{ }^{2}}{16}}+\sqrt{D_{6}(2)^{2}-\frac{r_{w}{ }^{2}}{4}}, \\
K_{x 8}=D_{6}(2)+2 \sqrt{D_{6}(2)^{2}-\frac{r_{w}^{2}}{16}}+2 \sqrt{D_{6}(2)^{2}-\frac{r_{w}{ }^{2}}{4}}, \\
K_{x 9}=r_{l}-\sqrt{D_{6}(2)^{2}-\frac{r_{w}{ }^{2}}{4}}, K_{x 10}=\sqrt{D_{6}(3)^{2}-\frac{r_{w}{ }^{2}}{4}} \\
K_{x 11}=3 \sqrt{D_{6}(3)^{2}-\frac{r_{w}{ }^{2}}{4}} .
\end{array}\right.
$$

Here the achievable distance of Type I $D_{6}(1)=\frac{1}{12} \sqrt{4 r_{l}^{2}+9 r_{w}^{2}}$, $D_{6}(2)$ of Type II is the smallest positive root of following equation:

$$
2 \sqrt{4 D_{6}(2)^{2}-r_{w}^{2}}+2 D_{6}(2)+\sqrt{4 D_{6}(2)^{2}-1 / 4 r_{w}^{2}}-r_{l}=0,
$$

and $D_{6}(3)=\frac{1}{30} \cdot\left(4 \sqrt{r_{l}^{2}+15 r_{w}^{2}}-r_{l}\right), D_{6}(4)=\frac{1}{12} \sqrt{r_{l}^{2}+36 r_{w}^{2}}$.

For other optimal placement cases with more than 6 APs, we can use the same way to first solve the thinnest covering problem, and then derive the optimal positions of APs in different types. Actually, for all cases reported on in later sections, deploying 6 APs provides more than sufficient performance gains and deploying additional APs provides little benefit.

In particular, from the optimal placement results of $1 \sim 6 \mathrm{APs}$, it is observed that there always exists one special deployment type that linearly arranges APs when the room is longer (i.e., $r_{l} / r_{w}$ is large), such as the results shown in Fig. 4 (b), Fig. 5 (c), Fig. 6 (d) and Fig.7 (d), thus we make an important conclusion: 


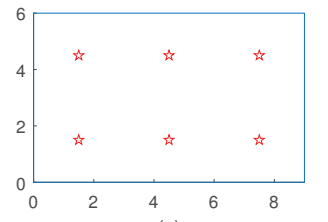

(a)

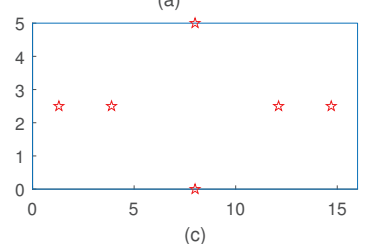

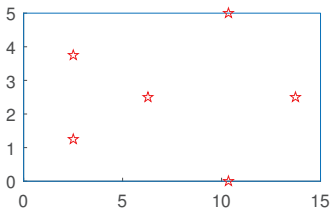

(b)

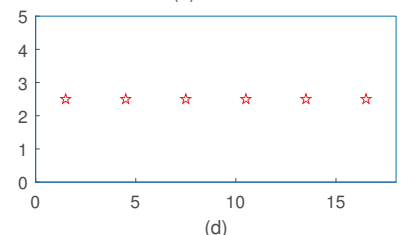

Figure 7: Optimal positions of 6 APs ((a) $r_{l}=9 \mathbf{m}, r_{w}=6 \mathbf{m}$; (b) $r_{l}=15 \mathbf{m}, r_{w}=5$; (c) $\left.r_{l}=16 \mathbf{m}, r_{w}=5 \mathbf{m} ;(\mathbf{d}) r_{l}=18 \mathbf{m}, r_{w}=5 \mathbf{m}\right)$.

Theorem 1 (Linear arrangement condition) In a specific room $\left(r_{l} \times r_{w} \times r_{h}\right)$ with $N$ APs, if $r_{l} / r_{w}>N / \sqrt{3}$, the optimal deployment method is to linearly arrange APs on the ceiling and along the center line of the shorter edge of room, where the optimal position of $i^{\text {th }} A P$ is $\left(\frac{(2 i-1) r_{l}}{2 N}, \frac{r_{w}}{2}, r_{h}\right)$.

Proof. According to the geometric analysis for thinnest covering of a longer rectangle, we can find the smallest radius of circles $r_{n}=d_{a c} \geq \frac{1}{2} \sqrt{\left(\frac{r_{l}}{N \cdot r_{w}}\right)^{2}+1}$, and the linear covering like in Fig. 4 (b) is the only arrangement that attains the lower bound of $r_{n}$ if $r_{l} / r_{w}>N / \sqrt{3}$. Then we can derive the $y$-axis coordinate of each AP must be in the middle of short edge of the room, and $x$-axis coordinate for $i^{t h} \mathrm{AP}$ along the longer edge of room is $\left[(2 i-1) r_{l}\right] / 2 N$. Since we adopt the ceiling-based APs to achieve better LOS performance (see Sec. 2), the $z$-axis of each AP is obtained as $r_{h}$.

\subsection{Analysis of multi-AP degree}

Based on the preceding theoretical analysis of multi-AP deployment in RORC scenarios, here we investigate the performance benefits of different numbers of APs.

First, we derive the expected LOS probability (ELP) as a metric for subsequent evaluation. Since a randomly-located client can be viewed as a random point distributed in a circle with a radius of $D_{n}$ (i.e., achievable distance $d_{a c}$ ), ELP of a random client and its connected AP is derived as:

$$
\begin{aligned}
E L P & =\int_{0}^{D_{n}} P_{L O S_{i, j}}(x) \cdot \frac{2 x}{D_{n}^{2}} d x \\
& =\frac{2 e^{-C_{2}}}{C_{1}^{2} \cdot D_{n}^{2}} \cdot\left(1-e^{-C_{1} \cdot D_{n}}-C_{1} \cdot D_{n} \cdot e^{-C_{1} \cdot D_{n}}\right)
\end{aligned}
$$

where $C_{1}=\frac{2 \varepsilon \cdot \lambda \cdot\left(\mu_{l}+\mu_{w}\right)}{\pi}, C_{2}=\varepsilon \cdot \lambda \cdot \mu_{l} \cdot \mu_{w}, P_{L O S_{i, j}}$ has been derived in Eq. (4), and $D_{n}$ is the maximum horizontal distance which can be obtained in Sec. 3.2 in terms of the number of APs $n$.

We evaluate the expected LOS probability with respect to the degree of APs and obstacle density. Fig.8 (a) shows the results in a $9 \mathrm{~m} \times 6 \mathrm{~m}$ room, and we find that deploying an odd number of APs brings smaller performance improvement than deploying an even number of APs, e.g., deploying 3 APs brings little performance increase compared with deploying $2 \mathrm{APs}$, but there is a relatively larger improvement with 4 APs instead of 3 APs. Besides, the performance gains become much smaller with higher numbers of APs, and this result is more obvious in Fig.8 (b) with a "longer" room $(18 \mathrm{~m} \times 5 \mathrm{~m})$, especially when the number of APs is larger than 5 , the sixth AP only brings a performance increase of less than $1 \%$.

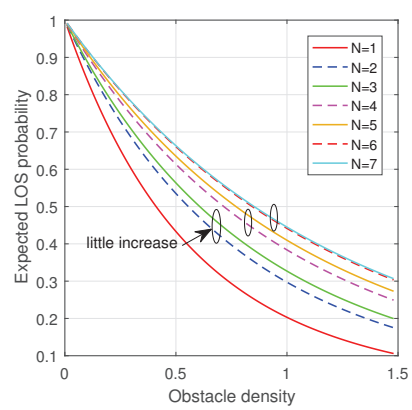

(a)

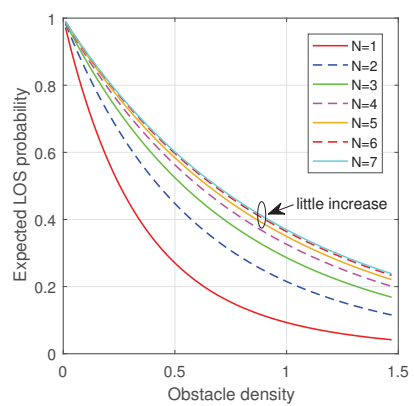

Figure 8: Excepted LOS probability vs. degree of AP and obstacle density.

In addition, we also investigate how room sizes affect the LOS performance. In Fig.9, with the specific obstacle density $(\lambda=0.4)$ and room's length-width ratio $\left(r_{l} / r_{w}=2.5\right)$, it shows that the ELP decreases as the room size increases with different numbers of APs, and this is because the maximal horizontal distance $\left(D_{n}\right)$ increases with a larger room, which results in a higher probability of experiencing blockage between an AP and its clients. On the other hand, as the number of APs increases with a specific room area, the performance improvement brought by higher numbers of APs is marginal, where the average performance increases over different room areas from $2^{\text {nd }}$ AP to $7^{\text {th }} \mathrm{AP}$ are $40.21 \%, 10.56 \%, 4.43 \%, 2.17 \%$, $1.43 \%$ and $1.06 \%$, respectively. Considering the cost of commercial mmWave AP devices, such as TP-Link Talon AD7200 Multi-Band Wi-Fi Router at $\$ 350$ [23], the second " $\$ 350$ " brings $40.21 \%$ potential performance increase, but the sixth or seventh " $\$ 350$ " only brings around $1 \%$ improvement, which also accords with the law of diminishing marginal utility. Therefore, we demonstrate that a number of APs of up to 5 offer higher relative performance gains, while improvements brought by higher degrees are marginal.

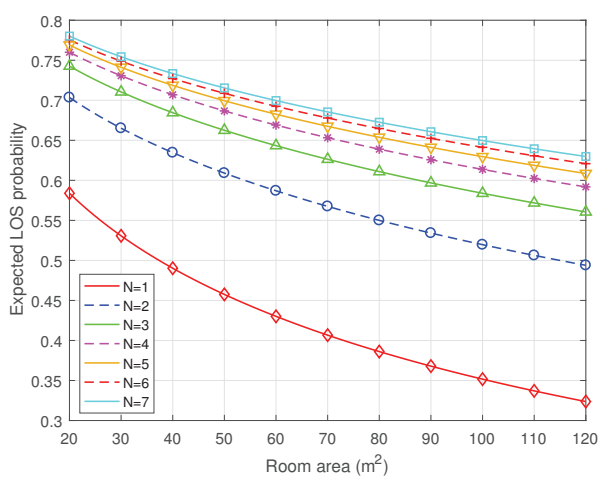

Figure 9: Excepted LOS probability vs. room size with different number of APs. 


\section{MULTI-AP PLACEMENT IN FORC SCENARIOS}

In this section, we consider another more realistic scenario, where the furniture such as tables, bookshelf or other objects with "obvious" heights ${ }^{1}$ has been placed in the room, which forms a FORC scenario. With these fixed and known obstacles in indoor settings, we investigate how to deploy the single or multiple APs that provides better LOS communications for random-located clients.

\subsection{Shadowing area minimization}

In the FORC scenario, to achieve better LOS performance with multiple APs, the problem becomes finding locations of ceilingmounted APs that can provide maximum effective LOS region for clients in the presence of known obstacles. In other words, the objective is to deploy APs so as to minimize the shadowing region (SR) caused by obstacles, so the formulated problem is described as:

$$
\underset{\text { Pos }}{\arg \min } \bigcup_{i \in O b s} S A_{i}, \forall \text { Pos } \in R m
$$

where $S A_{i}$ is the area of SR caused by the obstacle $i$, and Pos are the positions of APs.

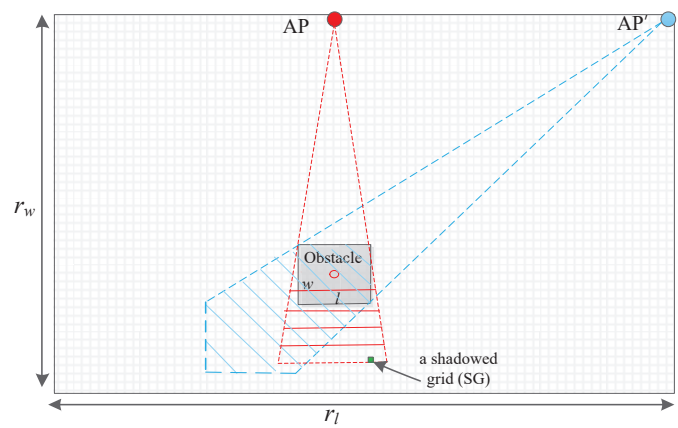

Figure 10: Top view of the SR caused by a single obstacle.

Fig. 10 shows an example of SR caused by a fixed obstacle, and the shape or size of SR is determined by several factors, such as the size of obstacle and the relative positions between AP and the obstacle. Here we make the assumption that obstacles in the room have only two orientations: $0^{\circ}$ and $90^{\circ}$, which means that the edges of obstacle will be parallel with the walls. This assumption is reasonable in practice since we typically deploy furniture in the same direction as one of the walls in the room.

To solve Eq. (15) and find the placement of APs, we introduce a grid-based shadowing search (GSS) method to calculate the shadowing area (SA), where the main idea is to first divide the rectangle (see Fig. 10) into a large number of small grids with side length ${ }^{2}$ $l_{g}$. Then, we find all shadowed grids (SG) whose center points fall in the shadowing region. After traversing all grid elements, SA is found as the cumulative area of shadowed grids. According to a geometric analysis, we determine that a grid element is shadowed if its center point exists in a shadowing polygon formed by an AP

${ }^{1}$ Here the object with the height larger than general height of client is considered as the obvious obstacle, since objects with very small heights will have no effect on LOS paths between ceiling-mounted APs and clients.

${ }^{2} \mathrm{~A}$ smaller $l_{g}$ provides more accurate results but has higher computational cost. and known obstacles. Due to space limitations, a detailed geometric analysis of this point-existent problem is not presented but can be found in our companion technical report [24].

\subsection{Single-AP single-obstacle case}

In this part, we start from a simple case where there are a single AP and one obstacle in the room. By using the GSS method to obtain SA in terms of different AP's positions, we can find the optimal AP's position which has minimum shadowing area $S A_{\min }$. Assuming the clients are randomly distributed in the room, the LOS probability can be derived as $S A_{\min } /\left(r_{w} \cdot r_{l}\right)$.

Fig. 11 shows an example with one fixed obstacle in a specific room $\left(12 m^{*} 8 m^{*} 3 m\right)$, and we observe that the optimal position of AP will be on one edge of the room (the red region shown in Fig. 11 (a)), and the corresponding LOS probabilities with different AP's positions are shown in Fig. 11 (b).
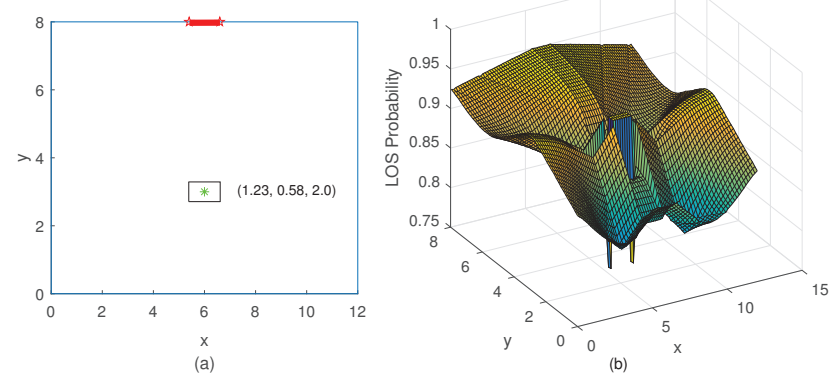

Figure 11: Optimal position of AP in a single-obstacle case (obstacle's height $h_{o}=2.0 \mathrm{~m}$ ).

When we consider a similar case but the obstacle has a lower height $\left(h_{o}=1.2 \mathrm{~m}\right)$, it is observed that the optimal position of $\mathrm{AP}$ is now above the obstacle (shown in Fig. 12). Therefore, we conclude that the obstacle's height has a significant impact on the optimal position of an AP, and deploying the AP on one edge of the room or directly above the obstacle achieves the best LOS performance in single-obstacle cases.
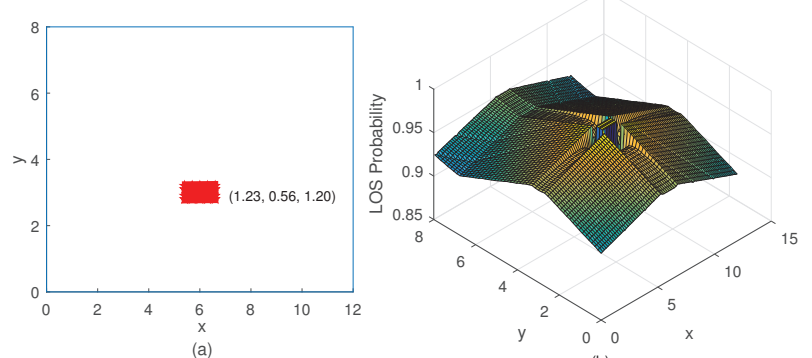

Figure 12: Optimal position of AP in a single-obstacle case (obstacle's height $h_{o}=1.2 \mathrm{~m}$ ).

\subsection{Single-AP multi-obstacle case}

Considering multi-obstacle cases with a single AP, one important step is to first find the union of SRs (i.e., without overlapping area) 
caused by different obstacles, and then the optimal placement of AP can be obtained by solving Eq. (15). Here, Algorithm 1 shows the method of calculating shadowing area with multiple obstacles for each AP's position. For every obstacle $i$, GSS method is conducted to get shadowed-grid set $S G_{i}$, which includes all grids shadowed by this obstacle (Line 3). Then, we start to check whether the grid in $S G_{i}$ has already been put in $S G_{n e w}$ (Lines 4-5), and if not, this new shadowed grid will be added in $S G_{n e w}$ (Lines 5-6). This step is used to eliminate the overlapping shadowing area caused by different obstacles. After traversing all known obstacles, the union of SRs is obtained (Line 10), and finally we find the optimal position of AP by solving Eq. (15).

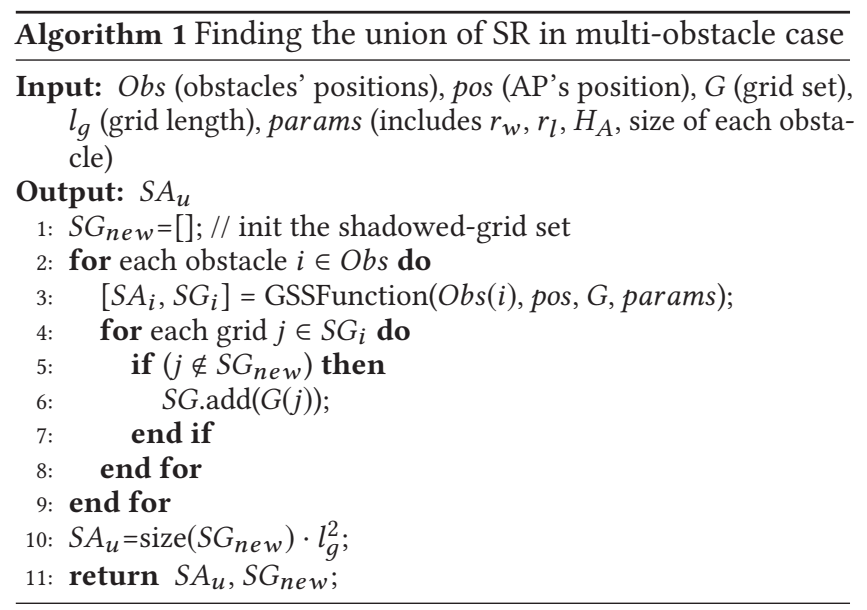

Fig. 13 shows two multi-obstacle cases with the optimal AP placement. In Fig. 13 (a), because four obstacles have the same sizes and are symmetrically located, there exist four equivalent positions (red stars) to optimally deploy the AP with minimum SR, but if all the heights of these obstacles are changed to $2.0 \mathrm{~m}$, we have only one optimal position of AP (blue star), which also implies that the heights of obstacles have a significant impact on optimal AP deployment. For another case shown in Fig. 13 (b), where multiple obstacles with different sizes are randomly distributed, only one optimal position of AP is found to achieve best LOS performance. From the results of these cases, we observe that the AP deployment in FORC scenarios is obviously different from that in RORC scenarios (placing AP in the center of the room), which means that the optimal AP placement is closely related to whether the information of obstacles is known or not.
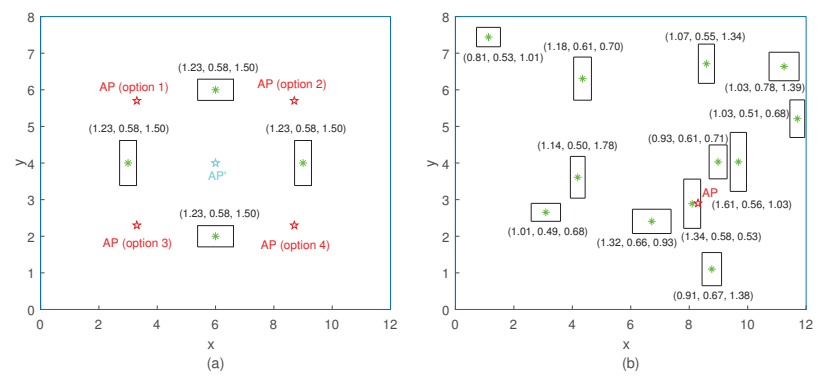

Figure 13: Optimal position of AP in multi-obstacle cases.

\subsection{Multi-AP multi-obstacle case}

In this part, we consider the multi-AP placement in FORC scenarios. Actually the optimal positions of multiple APs can be found by using the same method as in single-AP cases, but it is hard to solve Eq. (15) within a reasonable time when the number of APs is larger. Therefore, we propose a shadowing-elimination search (SES) algorithm to heuristically solve this multi-AP deployment problem.

Algorithm 2 summarizes the steps of the SES algorithm. First, considering all grids within the room, Algorithm 1 is conducted to get the union of shadowing area for each possible AP's position (Lines 3-5). Then, the optimal position of the first AP is found which has the minimum SR (Lines 6-7). Before starting to find the next AP's position, the grid set $G$ is updated as the shadowed-grid set of first AP (Line 8), which means that the following AP will be placed at the position that eliminates the most remaining SRs of the first AP. With this process, we can find all positions of $N$ APs with the minimum remaining $\mathrm{SR}$ of the first $\mathrm{AP}$ and achieve maximum coverage.

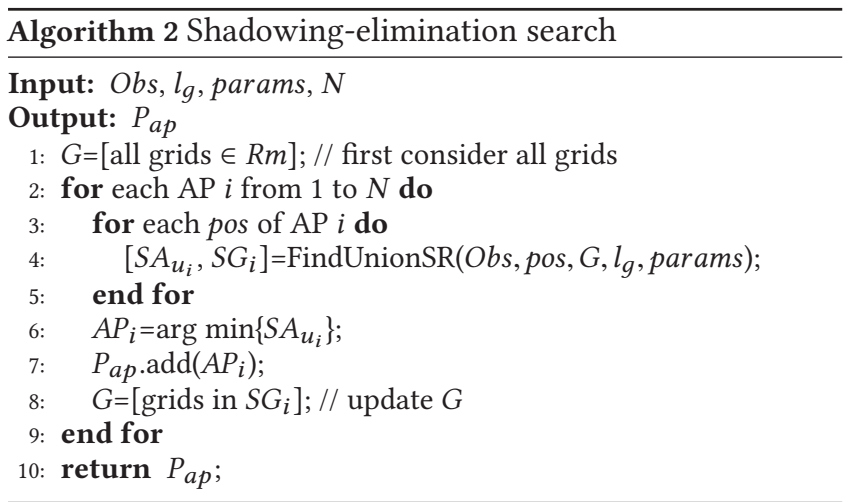

By running the SES algorithm, Fig 14 shows the placement of multiple APs in the same scenarios with Fig 13. By deploying 3 APs in each case, their remaining shadowing areas are $0.11 \mathrm{~m}^{2}$ and $0.56 \mathrm{~m}^{2}$, respectively.
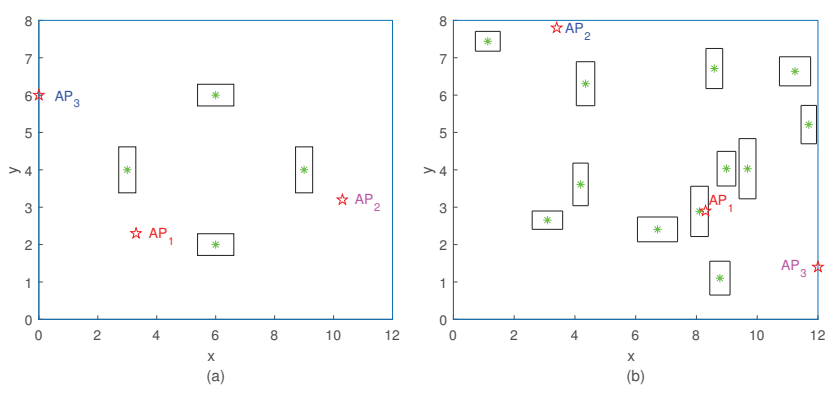

Figure 14: Multi-AP deployment in multi-obstacle cases.

\subsection{Blockage-free multi-AP mmWave WLANs}

As the density of AP increases, the remaining shadowing area will be entirely eliminated to achieve full coverage, i.e., the mmWave network scenario becomes blockage-free and randomly-located 
clients can always have LOS connections. In this part, we investigate how to place multiple APs to make the environment become blockage-free (shown in Algorithm 3). Based on the SES algorithm, we find the optimal position of the first AP (Lines 4-8), and then the grid set $G$ is updated for following shadowing elimination process (Line 9). Next, we compute the LOS probability $P_{L O S}$ which is related to the remaining shadowing area, and once all SR is eliminated such that $P_{L O S}$ becomes 1 , the whole process is terminated (Lines 10-13). Finally, the required number of APs and their respective positions are obtained (Line 16).

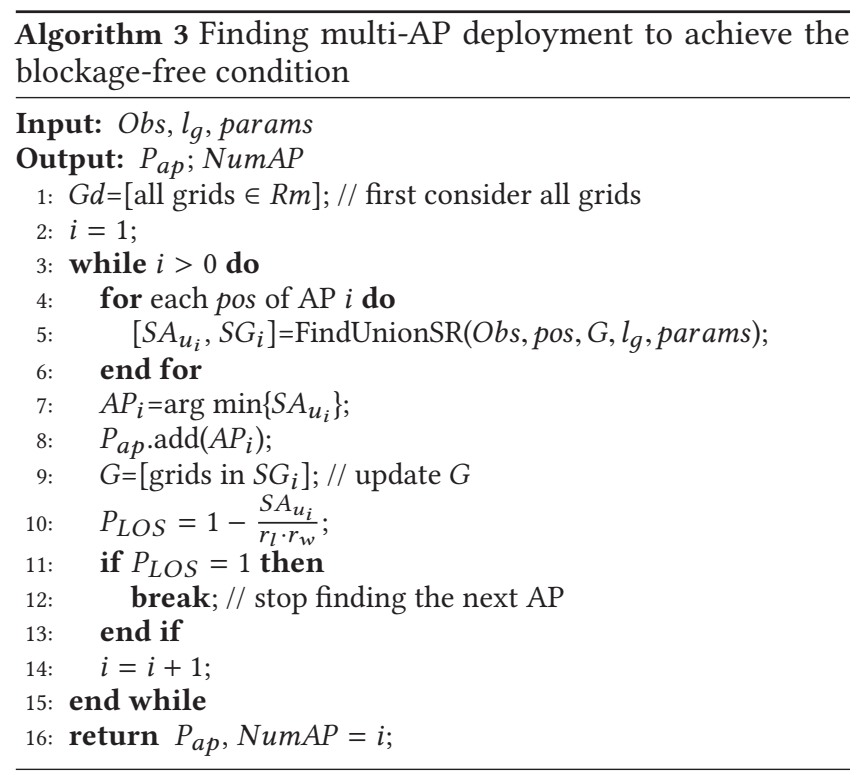

By running Algorithm 3, Fig. 15 shows two examples of placing multiple APs to achieve the blockage-free condition. It is observed that 3 and 4 APs are required in Fig. 15 (a) and (b), respectively, and with following multi-AP deployments in both cases, every client will have LOS communication because of the full coverage.
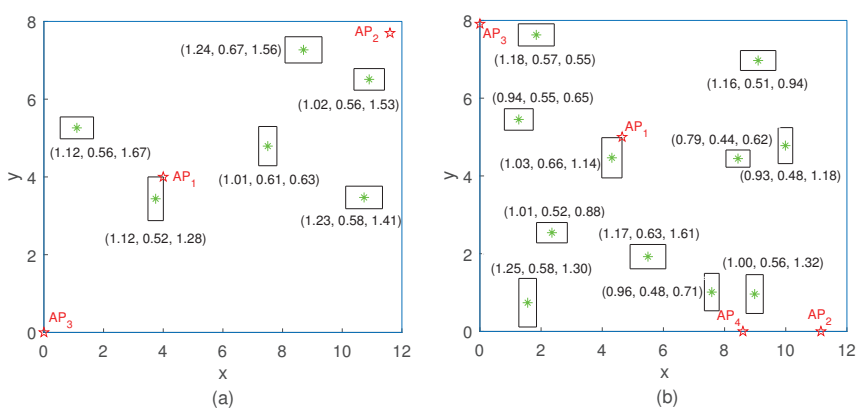

Figure 15: The deployment of multiple APs in blockage-free cases.

\section{PERFORMANCE STUDY}

In this section, we do the simulation-based analysis in multi-AP mmWave WLANs. First, the simulation platform based on ns-3 mmWave model is introduced. Then, we evaluate the network performance with proposed AP deployments in different scenarios.

\section{1 ns-3 mmWave indoor network model}

To incorporate the features of indoor configurations, we modified the ns-3 simulator based on IEEE 802.11ad protocol [25].

First, we implement the indoor scenario with an obstacle model that has following features: 1) obstacles are modeled as cuboids and placed on the floor; 2) the center of the obstacle follows a Poisson point process with a specific density; 3 ) the width and length of obstacle follow truncated normal distributions as $\mathrm{W} \sim \mathcal{T} \mathcal{N}(0.56$, $0.08,0.25,1.25)$ and $\mathrm{L} \sim \mathcal{T} \mathcal{N}(1.08,0.18,0.5,1.75) ; 4)$ the obstacle's height and orientation follows uniform distribution as $\mathrm{H} \sim \mathcal{U}(0.5$, 2) and $\Theta \sim \mathcal{U}(0, \pi)$. Besides, random-located client (i.e. wireless device) is viewed as a random point, and its height follows a uniform distribution as $\mathcal{U}(0.3,1.5)$. These parameters are derived by using a real-life lab environment as a guiding example.

Second, to build an accurate channel model for indoor mmWave communication, we collect 5 sets of experimental estimations of path loss model (including the path loss exponent and distribution of fast fading), where all experiments are performed with LOS connections in the lab environment [26].

\subsection{Performance in RORC scenarios}

In this part, we evaluate network performance with optimal multiAP placement in RORC scenarios, and all simulation parameters of mmWave system are adopted from Sec. 5.1. Here LOS probability and aggregate throughput are considered as main metrics for evaluation. First, considering a specific room where $r_{l}=12 \mathrm{~m}, r_{w}=8 \mathrm{~m}$, we compare the network performance of proposed multi-AP deployment (see Sec. 3.2) against that of three common deployment methods, which are 1) random deployment, 2) linear arrangement where APs are deployed according to Theorem 1, and 3) edged deployment where AP are randomly placed on the edges of room. With a specific obstacle density $\lambda=0.3$, Fig. 16 (a) and (b) shows the LOS probability and throughput performance over 1 6 APs, respectively. It is observed that proposed optimal deployment outperforms other three deployment methods. Compared with the linear deployment method, the optimal placement provides the obvious improvement on LOS probability and throughput when the number of APs increases. The random deployment shows worse performance when deploying fewer number of APs, and the edged deployment always provides worst performance due to its limited coverage.
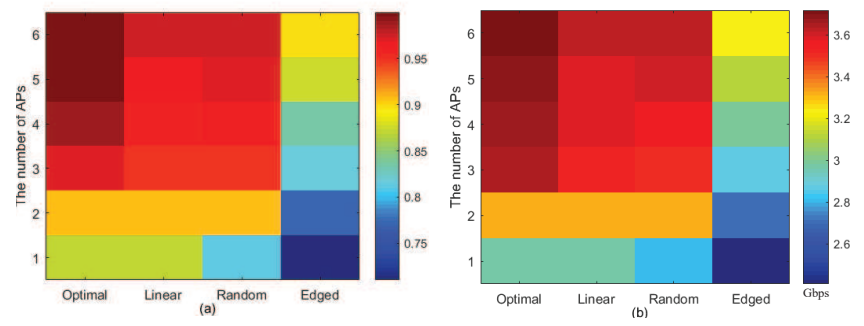

Figure 16: Deployment methods comparisons.

Second, with optimal placement of single AP or multiple APs in the room, we evaluate the network performance with different obstacle densities. In Fig. 17 (a), we observe that the LOS probability decreases when the obstacle density becomes higher due to server 
potential blockage effects, and with higher degree of deployed APs, both LOS and throughput performance (shown in Fig. 17 (b)) will be improved but the increase becomes marginal (less than 1\%) when deploying 6 APs, and this result confirms our theoretical analysis in Sec. 3.3. On the other hand, we can see that the throughput is always consistent with LOS performance, which confirms that LOS is a critical requirement for indoor mmWave communication. Thus, in what follows, we focus solely on LOS performance.
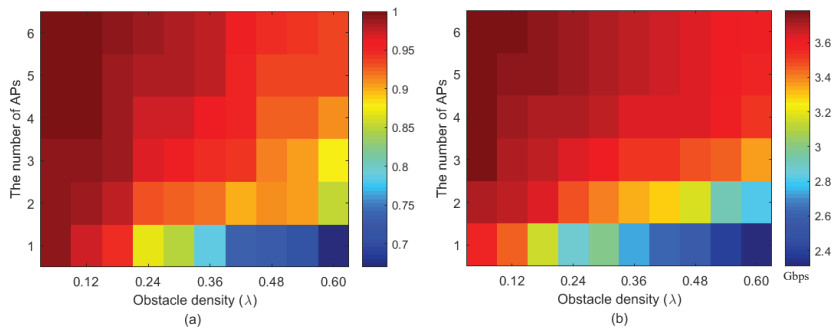

Figure 17: Network performance vs. obstacle density.

\subsection{Performance in FORC scenarios}

In this part, we evaluate the all-client LOS rate (ALR) in an indoor scenario shown in Fig. 15 (b), where 11 fixed obstacles are deployed. With randomly located clients, we conduct hundreds of simulations, and ALR is 1 if all clients have LOS connections in each case, otherwise it is 0 . By using the SES algorithm to determine multi-AP placement in this scenario, Fig.18 shows the ALR performance with different numbers of APs and random clients. First, we can see that ALR achieves over $90 \%$ when deploying 3 APs even with 15 randomly located clients, which is a huge improvement compared to the single AP case. Second, we know that full coverage will be achieved with deployment of 4 APs (shown in Fig. 15 (b)), and here Fig. 18 indeed shows ALR is over $99.99 \%$ with 4 APs deployed, which validates our SES algorithm since all shadowing areas are almost eliminated with generated AP placement.

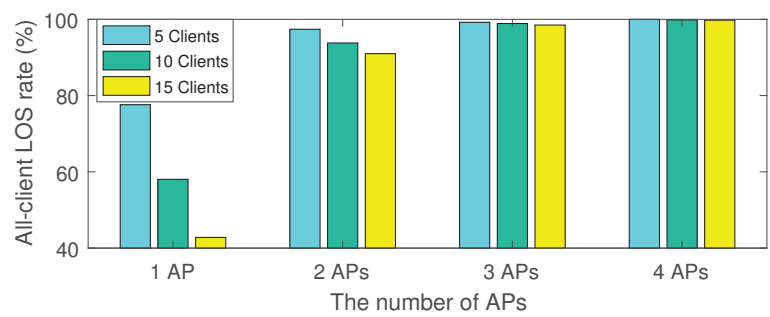

Figure 18: All-client LOS rate vs. number of APs.

In addition, we compare the LOS performance between the optimal multi-AP placement (by solving Eq. (15) in a brute-force method) and the placement generated by the SES algorithm (referred to as SES deployment). To achieve a blockage-free condition, Fig. 19 shows the optimal result and SES result. Through the ALR comparison (shown in Fig. 20), the performance of SES deployment is very close to that of optimal deployment with same number of APs, and even though one more AP is required by SES algorithm to entirely eliminate shadowing region, but actually after deploying the third AP shown in Fig 19 (b), ALR is above $98.5 \%$ without the $4^{\text {th }}$
$\mathrm{AP}$, and the remaining shadowing area is only $0.73 \mathrm{~m}^{2}$ (shown in Tab. 1), which is very close to full coverage. Therefore, the proposed SES algorithm provides near-optimal multi-AP deployment in the evaluated scenarios. In addition, the SES algorithm has obviously lower time cost to generate results as shown in Tab. 1 for this case.
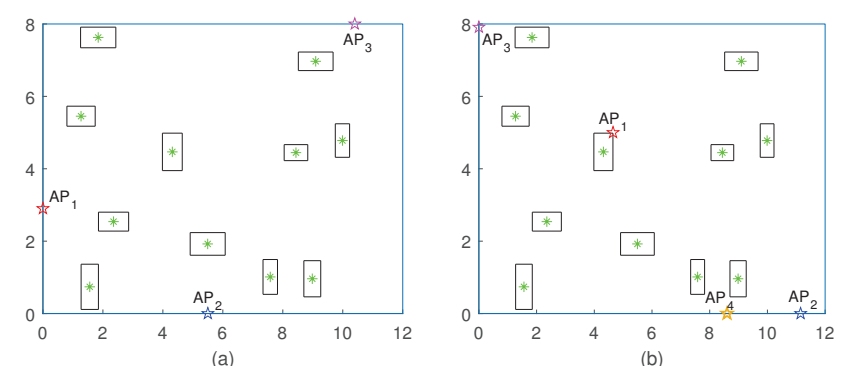

Figure 19: Multi-AP placements for blockage-free case. ((a) Optimal solution, (b) Solution from SES algorithm)

Table 1: The remaining shadowing area (RSA) and time cost with SES algorithm and optimal (brute-force) solution.

\begin{tabular}{|c|c|c|c|c||c|}
\hline No. AP & 1 & 2 & 3 & 4 & Time cost $(\mathrm{hr})$ \\
\hline Opt's RSA $\left(\mathrm{m}^{2}\right)$ & 16.13 & 1.43 & 0 & N/A & $\sim 85$ \\
\hline SES’s RSA $\left(\mathrm{m}^{2}\right)$ & 16.13 & 3.56 & 0.73 & 0 & 0.75 \\
\hline
\end{tabular}

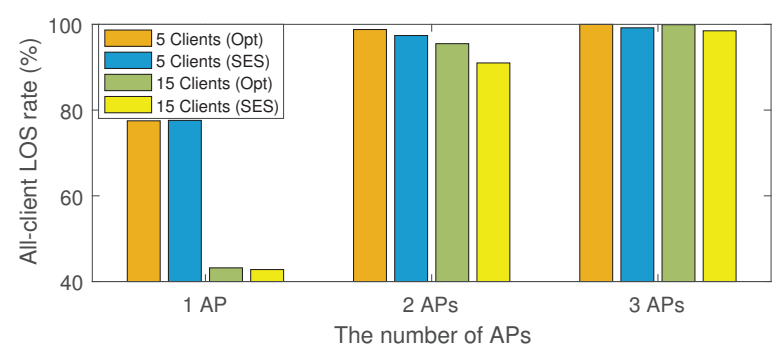

Figure 20: ALR comparison between optimal and SES results.

Considering the computation efficiency of proposed algorithm, we also compare the time costs (TC) of brute-force (BF) method and SES algorithm in terms of different number of fixed obstacles. As shown in Tab. 2, the time cost of generating optimal solution with $\mathrm{BF}$ method has a nearly exponential increase when there exists over 4 obstacle in FORC scenarios, but the time cost of SES algorithm has only a gently increase as the number of obstacles increase, which shows an absolute advantage in computation efficiency with multiple fixed obstacles.

Table 2: The time cost comparisons with SES algorithm and optimal (brute-force) method.

\begin{tabular}{|c|c|c|c|c|c|}
\hline No. of Obstacles & 2 & 4 & 6 & 8 & 10 \\
\hline BF's TC $(\mathrm{hr})$ & 0.23 & 2.57 & 13.21 & 36.32 & 82.35 \\
\hline SES's TC $(\mathrm{hr})$ & 0.18 & 0.26 & 0.48 & 0.59 & 0.68 \\
\hline
\end{tabular}


Finally, we compare the number of required APs to achieve blockage-free conditions with the SES algorithm and the optimal solution. Since it is too time consuming to get optimal results with a large number of obstacles, here we considered cases with a few obstacles, and then evaluated the average number of APs to achieve full coverage. Fig. 21 shows that the number of required APs with SES algorithm is close to optimal, and as the obstacle scale increases, the gap between SES and optimal becomes smaller, which validates the performance of the SES algorithm.

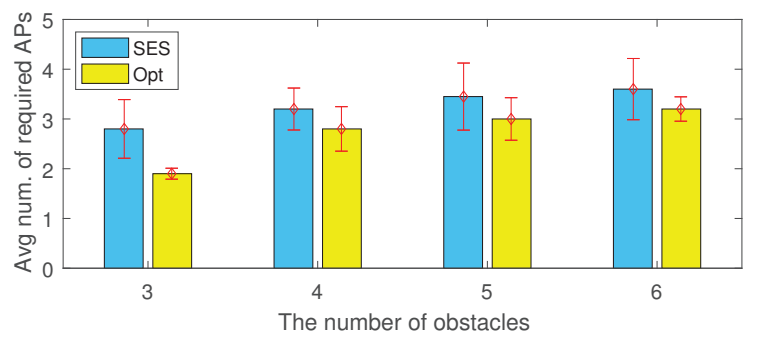

Figure 21: Avg. no. of required APs vs. no. of obstacles.

\section{DISCUSSION}

Our results can be directly used in practice for network deployment in several ways. If APs are pre-deployed before a room is furnished, or if it is expected that furniture will be moved frequently and moving AP locations is difficult, then APs should be deployed according to the optimal locations derived from the thinnest covering problem solution in the RORC scenario. However, if APs can be positioned after furniture locations are known, then they should be deployed at the locations provided by the SES algorithm for the multi-AP, multi-obstacle FORC scenario.

In addition, the proposed analytical model and algorithms in this paper can also generate AP placements for non-rectangular rooms, if those rooms are combinations of several rectangular rooms. For example, considering an "L-shaped" room, we can divide it into two rectangular rooms, and generate the optimal (near-optimal) positions of APs for each of them separately.

On the other hand, considering the cost of multiple mmWave AP devices used in indoor scenarios, an alternative is to deploy a single AP and multiple low-cost relays to extend the AP's coverage. In this case, the only additional step is to select an appropriate position for the unique AP among all generated optimal locations. One strategy would be to place the $\mathrm{AP}$ at the position with best coverage. For example, in RORC scenarios, we choose the optimal location that has the smallest achievable distance $d_{a c}$, while in FORC scenarios, the AP could be deployed at the position that is the first one generated by the SES algorithm.

\section{CONCLUSION}

In this paper, we considered coverage and deployment issues in multi-AP mmWave WLANs. Based on our analytic model, the optimal locations of multiple APs were derived in RORC scenarios, and we showed that deploying up to 5 APs provides the highest performance gains. In the FORC scenario, the shadowing-elimination search algorithm was proposed to determine the placement of APs, and full coverage is achieved with enough APs. Through ns-3 IEEE 802.11ad simulations at $60 \mathrm{GHz}$, the network performance of proposed AP deployments is shown to be always superior to that of other common placement methods. Besides, we also validate that the proposed SES algorithm can generate near-optimal placement of APs, which provides desirable network performance for clients.

\section{ACKNOWLEDGMENTS}

This research was supported in part by the National Science Foundation through Award CNS-1813242.

\section{REFERENCES}

[1] Xiong Wang, Linghe Kong, Fanxin Kong, Fudong Qiu, Mingyu Xia, Shlomi Arnon, and Guihai Chen. Millimeter wave communication: A comprehensive survey. IEEE Communications Surveys \& Tutorials, 20(3):1616-1653, 2018.

[2] Theodore S Rappaport et al. Millimeter wave mobile communications for $5 \mathrm{~g}$ cellular: It will work! IEEE access, 1, 2013.

[3] Ieee std 802.11ad-2012, 2012. URL: https://ieeexplore.ieee.org/stamp/stamp.jsp? arnumber $=6392842$

[4] Thomas Nitsche et al. Ieee 802.11 ad: directional $60 \mathrm{ghz}$ communication for multi-gigabit-per-second wi-fi. IEEE Communications Magazine, 2014

[5] Yuchen Liu and Douglas M Blough. Analysis of blockage effects on roadside relay-assisted mmwave backhaul networks. In 2019 IEEE International Conference on Communications (ICC), pages 1-7. IEEE, 2019.

[6] Sumit Singh et al. Blockage and directivity in $60 \mathrm{ghz}$ wireless personal area networks: From cross-layer model to multihop mac design. Fournal on Selected Areas in Communications, 27(8):1400-1413, 2009.

[7] Minyoung Park and Helen K Pan. A spatial diversity technique for ieee 802.11 ad wlan in $60 \mathrm{ghz}$ band. IEEE communications letters, 16(8):1260-1262, 2012.

[8] Zulkuf Genc et al. Robust $60 \mathrm{ghz}$ indoor connectivity: Is it possible with reflections? In 2010 IEEE 71st vehicular technology conference. IEEE, 2010.

[9] Xia Zhou et al. Mirror mirror on the ceiling: Flexible wireless links for data centers. ACM SIGCOMM Computer Communication Review, 42(4):443-454, 2012.

[10] Mathew K Samimi et al. Characterization of the $28 \mathrm{ghz}$ millimeter-wave dense urban channel for future $5 \mathrm{~g}$ mobile cellular. NYU Wireless TR, 1, 2014.

[11] Yong Niu et al. Blockage robust and efficient scheduling for directional mmwave wpans. IEEE Transactions on Vehicular Technology, 64(2):728-742, 2015.

[12] Kan Song et al. A fast relay selection algorithm over $60 \mathrm{ghz} \mathrm{mm}$-wave systems. In 2013 15th IEEE International Conference on Communication Technology, 2013.

[13] Yuchen Liu et al. Blockage avoidance in relay paths for roadside mmwave backhaul networks. In 2018 IEEE 29th Annual International Symposium on Personal, Indoor and Mobile Radio Communications (PIMRC), pages 1-7. IEEE, 2018.

[14] Fan Zhou et al. Making the right connections: Multi-ap association and flow control in $60 \mathrm{ghz}$ band. In IEEE INFOCOM 2018-IEEE Conference on Computer Communications, pages 1214-1222. IEEE, 2018.

[15] Xiaoqi Qin et al. On ap assignment and transmission scheduling for multi-ap $60 \mathrm{ghz}$ wlan. In 2017 IEEE 14th International Conference on Mobile Ad Hoc and Sensor Systems (MASS), pages 189-197. IEEE, 2017.

[16] Sanjib Sur et al. Towards scalable and ubiquitous millimeter-wave wireless networks. In Proceedings of the 24th Annual International Conference on Mobile Computing and Networking, pages 257-271. ACM, 2018.

[17] Zhicheng Yang et al. Sense and deploy: Blockage-aware deployment of reliable $60 \mathrm{ghz}$ mmwave wlans. In 2018 IEEE 15th International Conference on Mobile Ad Hoc and Sensor Systems (MASS), pages 397-405. IEEE, 2018.

[18] Wei Li et al. Analysis of base station deployment impact on los probability model for $5 \mathrm{~g}$ indoor scenario. In 2017 IEEE/CIC International Conference on Communications in China (ICCC), pages 1-5. IEEE, 2017.

[19] Richard Cowan. Objects arranged randomly in space: an accessible theory. Advances in Applied Probability, 21(3):543-569, 1989.

[20] Tianyang Bai et al. Analysis of blockage effects on urban cellular networks. IEEE Transactions on Wireless Communications, 13(9):5070-5083, 2014.

[21] Aladár Heppes and Hans Melissen. Covering a rectangle with equal circles. Periodica Mathematica Hungarica, 34(1-2):65-81, 1997.

[22] Johannes Bernardus Marinus Melissen et al. Covering a rectangle with six and seven circles. Discrete Applied Mathematics, 99(1-3):149-156, 2000.

[23] Tp-link talon ad7200 multi-band wi-fi router, 2016. URL: https://www.pcmag. com/review/347405/tp-link-talon-ad7200-multi-band-wi-fi-router.

[24] Yuchen Liu and Douglas M Blough. Technical report for grid-based shadowing search method. (available to reviewers at: http://blough.ece.gatech.edu/TR-MultiAP-indoor.pdf).

[25] Hany Assasa and Joerg Widmer. Implementation and evaluation of a wlan ieee 802.11 ad model in ns-3. In Proceedings of the Workshop on ns-3. ACM, 2016.

[26] Peter FM Smulders. Statistical characterization of 60-ghz indoor radio channels. IEEE Transactions on Antennas and Propagation, 57(10):2820-2829, 2009. 